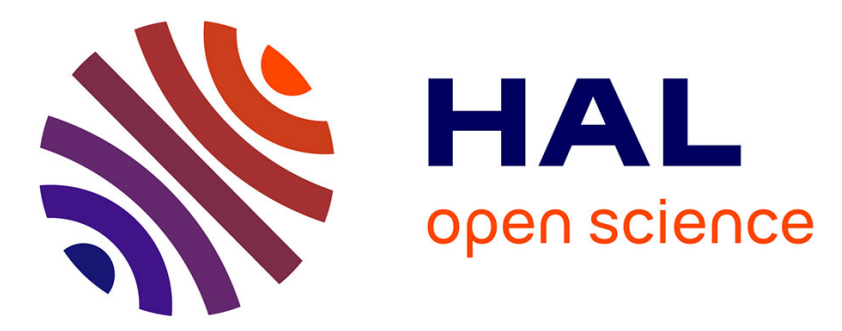

\title{
A Synergetic Brain-Machine Interfacing Paradigm for Multi-DOF Robot Control
}

Saugat Bhattacharyya, Shingo Shimoda, Mitsuhiro Hayashibe

\section{To cite this version:}

Saugat Bhattacharyya, Shingo Shimoda, Mitsuhiro Hayashibe. A Synergetic Brain-Machine Interfacing Paradigm for Multi-DOF Robot Control. IEEE Transactions on Systems, Man and Cybernetics, Part A: Systems and Humans, 2016, 46 (7), pp.957-968. 10.1109/TSMC.2016.2560532 . lirmm01347425

\section{HAL Id: lirmm-01347425 \\ https://hal-lirmm.ccsd.cnrs.fr/lirmm-01347425}

Submitted on 21 Jul 2016

HAL is a multi-disciplinary open access archive for the deposit and dissemination of scientific research documents, whether they are published or not. The documents may come from teaching and research institutions in France or abroad, or from public or private research centers.
L'archive ouverte pluridisciplinaire HAL, est destinée au dépôt et à la diffusion de documents scientifiques de niveau recherche, publiés ou non, émanant des établissements d'enseignement et de recherche français ou étrangers, des laboratoires publics ou privés. 


\title{
A Synergetic Brain-Machine Interfacing Paradigm for Multi-DOF Robot Control
}

\author{
Saugat Bhattacharyya, Shingo Shimoda, and Mitsuhiro Hayashibe, Senior Member, IEEE
}

\begin{abstract}
This paper proposes a novel brain-machine interfacing (BMI) paradigm for control of a multijoint redundant robot system. Here, the user would determine the direction of end-point movement of a 3-degrees of freedom (DOF) robot arm using motor imagery electroencephalography signal with coadaptive decoder (adaptivity between the user and the decoder) while a synergetic motor learning algorithm manages a peripheral redundancy in multi-DOF joints toward energy optimality through tacit learning. As in human motor control, torque control paradigm is employed for a robot to be adaptive to the given physical environment. The dynamic condition of the robot arm is taken into consideration by the learning algorithm. Thus, the user needs to only think about the end-point movement of the robot arm, which allows simultaneous multijoints control by BMI. The support vector machine-based decoder designed in this paper is adaptive to the changing mental state of the user. Online experiments reveals that the users successfully reach their targets with an average decoder accuracy of over $75 \%$ in different end-point load conditions.
\end{abstract}

Index Terms-Brain-machine interfacing (BMI), co-adaptive decoder, joint redundancy, multijoint robot, synergetic learning control, tacit learning.

\section{INTRODUCTION}

A S OF today, brain-machine interfacing (BMI) [or braincomputer interfacing (BCI)] is one of the fastest growing areas of research that provides a unique course of communication between a human and a machine (or device) without any neuro-muscular intervention [1]. BMI was initially conceived to provide rehabilitative and assistive solutions [2], [3] to patients suffering from neuromuscular degenerative diseases, such as amyotropic lateral sclerosis, cervical spinal injury, paralysis, or amputee [4]. But in recent years, potential applications in fields of communication [5], [6], military use [7], virtual reality [8], [9] and gaming [10], [11] has

Manuscript received October 22, 2015; revised December 17, 2015; accepted March 7, 2016. Date of publication May 26, 2016; date of current version June 14, 2016. This work was supported by the Erasmus Mundus Action 2 project for Lot 11-Svaagata.eu:India through European Commission (ref.nr. Agreement Number: 2012-2648/001-001-EM Action 2-Partnerships). This paper was recommended by Associate Editor Z. Li.

S. Bhattacharyya is with the INRIA-LIRMM, University of Montpellier, Montpellier 34095, France (e-mail: saugatbhattacharyya@live.com).

S. Shimoda is with the Brain Science Institute-Toyota Collaboration Center, RIKEN, Nagoya 2271-130, Japan.

M. Hayashibe is with the INRIA-LIRMM, University of Montpellier, Montpellier, France, and also with the Brain Science Institute-Toyota Collaboration Center, RIKEN, Nagoya, Japan (e-mail: hayashibe@lirmm.fr).

Color versions of one or more of the figures in this paper are available online at http://ieeexplore.ieee.org.

Digital Object Identifier 10.1109/TSMC.2016.2560532 widened its materiality across different domain other than rehabilitation.

A BMI system relies on tools from digital signal processing and machine learning to identify and predict the cognitive state of the user from their corresponding brain signals [4]. The brain signals are recorded either invasively or noninvasively [12]. Although invasive means of acquisition provides better performance in terms of accuracy and precision, noninvasive means are widely used by most BMI/BCI researchers for their simplicity in user interface. The most widely used noninvasive recording technique is electroencephalography (EEG), where the signals are recorded by electrodes placed on the scalp, because it is inexpensive, portable, easily available and has high temporal resolution [4], [13].

Depending on the nature of the experiment, the acquired EEG is found to have specific signal characteristics. Signals acquired during movement related planning, imagination or execution [motor imagery (MI)] is identified by a decrease in spatio-spectral power [termed as event-related desynchronization (ERD)] followed by an increase in power [termed as event-related synchronization (ERS)] [14], [15]. Researchers have widely used the changing patterns of ERD/ERS patterns for different MI tasks [such as left (or right) hand $\mathrm{MI}$ ] to generate commands necessary to drive a peripheral device such as mobile [16], [17] or humanoid robots [18], wheelchairs [19] and navigation in virtual reality [8], and gaming [20] environment.

Even after such advances of EEG-BMI in control applications, it still has not been used in real world applications (except simple discrete selection task) because of certain issues inherent in the signal. EEG signals are nonstationary, nonlinear, non-Gaussian, and highly variable in nature [1], [15], because the recordings on different days or different times of the same day exhibit high variability of the signal. This phenomena usually occurs due to shifts in electrode positions between sessions or changes in the electrochemical properties of the electrodes. Another issue that arises from EEG is the noisy and low resolution signals recorded from the scalp, which in actuality is the nonlinear superposition of electrical activity of a large population of neurons. This masks the underlying neural pattern of interest and restricts their detection. Even the current mental state of the user may affect the quality of the signal [1], [21]. To address these problems, a practical BMI system should continuously track the changing EEG patterns of the user in order to obtain a good performance.

2168-2216 (c) 2016 IEEE. Translations and content mining are permitted for academic research only. Personal use is also permitted, but republication/ redistribution requires IEEE permission. See http://www.ieee.org/publications_standards/publications/rights/index.html for more information. 
Study on the co-adaptivity of the user with the BMI system is an active area of research and to date, there is not much literature available on auto-adaptive and autocalibrated approaches. In current co-adaptive approaches [1], [21], [22], the system is initially trained to previous data, which is used for initial training of the decoder. Then, data collected from subsequent sessions are directly included into the system, where the decoder is retrained and updated. The performance of the user is displayed visually after the task, which allows the user to train him/herself. DiGiovanna et al. [23] used reinforcement learning to develop an intelligent BMI control agent that works in synergy with the BMI user and both the system co-adapts and continuously learns from the environment. The model was tested on rats and each subject co-adapted with BMI control system significantly to control a prosthesis. Recently, Bryan et al. [24] have devised a new approach to BCI, which employs partially observable Markov decision processes to handle the uncertainty of the EEG and achieve coadaptivity. Their approach allowed the system to make online improvements to its behavior by adjusting itself to the user's changing circumstances.

Till now, a discussion on co-adaptation learning based on both user and BMI system has been provided. Based on these approaches, it is possible to control a robot (or prosthetic) limb using MI BMI commands (like, left hand, and right hand). But, control of multijoint robot involves redundancy management issues in simultaneous multijoint control which is an open problem in this area. To control multijoint robot using the current control paradigms of BMI, one may need to control each individual joints separately in step-by-step manner to complete a task [15], [19], [25]. Such movement of the robot arm is not similar to human motor control, and is tedious to the user. As a result, such control techniques do not provide a practical solution and are far from natural human limb coordination since it is ideal to employ a control framework which allows users to drive BMI-driven robot as a third arm by his feeling. By following a human-like synergetic motor control framework, one may obtain optimal BMI control solutions in multidegrees of freedom (DOF) arm which is similar to the case in actual human motor control [26]. For instance, when we try to get a glass of water, we imagine mainly about an end-point task itself in reaching motion than imagining about individual joint angle trajectory. It is more natural to imagine such end-point intentions and it can be obtained even through noninvasive BMI using superficial cortical level signals. Redundant peripheral joint control should be managed at different level as it is normally managed in cerebellum level in human motor control.

In this paper, we propose a novel BMI paradigm, which is a combination of a co-adaptive EEG decoder, which adapts the decoder to the current mental state of the user while he/she observes a feedback (FB) [22] (in this paper, the motion of the robot), and synergetic motor learning scheme [26], to control the movements of a multijoint redundant robot driven by torque control. The synergetic learning controller takes on a role of functionality of cerebellum to optimize the peripheral motor coordination taking into account the given dynamic environment. Torque control scheme is preferred in humanoid robotics as it provides environmental compliance for humanrobot interaction [27]. Regarding motor intention in cortical level, the decoder distinguishes between left and right MI EEG to move a 3-DOF robot up and down toward a given target. As a result, by blending the cortical signal level learning paradigm of the BMI-user system and the peripheral motor learning paradigm, we have attempted to simplify the BMI control of a multijoint robot in a fashion similar to the situation where we control a human limb naturally. As it is first trial and report of this new BMI paradigm on redundant robot, a relatively simple task focusing on the joint level handling is employed in this paper. However, this paper first deals with tridirectional adaptation in BMI. In addition to the so-called bilateral adaptation between human physiological signal changes and its adaptive decoding, the third adaptation in peripheral motor control is integrated to deal with redundant arm coordination.

The rest of this paper is organized as follows: Section II describes the synergetic BMI control paradigm proposed in this paper. This section also provides information on the experimental setup. The results of the experiments are presented and discussed in Section III. Section IV presents a comparative discussion of this paper followed by concluding remarks on Section V.

\section{Principles And Methodology}

\section{A. Synergetic BMI Control Scheme}

It is known that human beings do not perform the joint actions of compound movements consciously. Movements are generally controlled by a subconscious mental subroutine and thus, can be considered as automatic in nature [28]. While learning a new movement the mental activity shifts from the foreground mental routine to the background subconscious one. Thach [28] and Wolpert et al. [29] suggested that training of skilled movements in the human brain starts as a conscious act in the cerebral cortex. But on gradual and repetitive trials of the same movement, the cerebellum begins to take control of the task by recognizing the relation to each segment of consciously initiated movement. Finally, the cerebullum attains control over the entire process and by a mere trigger from the cerebrum, it can execute the entire movement without any conscious effort [28]-[30]. The multijoint human motor system requires to handle complex interaction torques which is compensated by predictive motor control located within the cerebellar cortex. Sensory information on the early phases of the movement enters the cerebellum and triggers the memory related to the optimal joint torque. As a result, motor learning and control are executed flawlessly and are easily adapted to the ever-changing environment and newly generated goals. The aim of BMI control of a prosthetic or robotic limb is to allow seamless human-like movement but to date, they incur joint redundancy issues during movement tasks. To solve this problem, one needs to include a learning controller to manage peripheral drive for a multijoint system to allow an optimal human-like movement of the limb.

Several models have been formulated to deal with the redundancy issues in the past and such models are generally defined as "minimum $X$," where $X$ is jerk [31], torque 


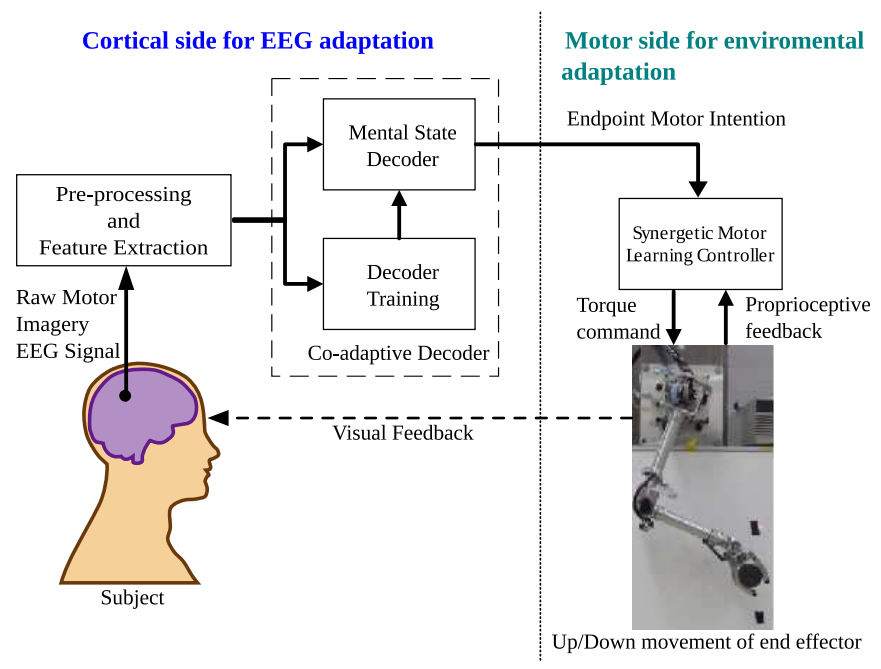

Fig. 1. BMI paradigm employed in this paper for simultaneous control of multi-DOFs robot using adaptive left-right MI decoder and synergetic motor learning for peripheric joint redundancy management. The black dots indicate the targets for the subjects in the vertical plane.

changes [32], motor command [33], and energy consumption [34]. Researchers basically assume the use of a physical inverse dynamical model [35] or approximation-based models [36]. Hayashibe and Shimoda [26] have proposed an optimal method for multijoint redundancy management using tacit learning scheme. This technique optimizes the multijoint problem without any prior knowledge of the system dynamics by using the task space error. Phenomenological optimal solutions can be generated without using so-called mathematical optimization process. In this paper, we have adopted this synergetic learning control technique for the peripheral multijoint management of a 3-DOF robotic arm.

The details of the online BMI control paradigm, shown in Fig. 1, are as follows. The participant observes the current position of the end-effector of the robot and attempts to generate the required MI signal. The process involves filtering and extracting features from the raw EEG signal. Then, the features are fed as inputs to the decoder to identify the MI state (left/right MI). The decoded output is then transmitted to the robot as commands to move it up or down in the vertical plane. Prior to the onset of the online task, the robot is trained to its dynamic environment using a tacit learning approach [37] for a fixed period of $70 \mathrm{~s}$. In this paper, the load carried by the robot is treated as the environmental change along with segmental inertial configuration changes. As a result, the movement of the joints of the robot adapts to the changing load. To make the decoder co-adaptable to the changing brain state of the subject, we measure the posterior probability $(P)$ of each incoming event. If $P$ fulfills the required conditions of the system then it is included in the training dataset with a higher weight than the older data, while the oldest data is removed from the dataset and the decoder is retrained online. If $P$ does not fulfill the conditions, then we reject the incoming data and the decoder does not need to be retrained. This step is included to change the learning of the decoder with the current mental state of the subject.

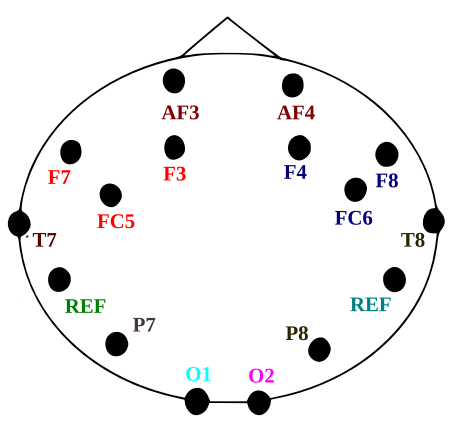

Fig. 2. Standard 10-20 representation of the electrodes present in an Emotiv headset.

Our proposed scheme adapts at different stages. First, the decoder/ classifier is designed to continuously adapt to the changing brain signal, while the subject simultaneously observes the movement of the robot. Second, the peripheral motor controller is adaptable to the given physical environment. Because of the two adaptive function, the subject is free to control the robot arm without burdening himself to control complex joint management. Hence, here we have proposed a tridirectional form of adaptation (user-decoder-robot).

\section{B. Experiment Description}

1) Subjects and Data Acquisition: The EEG in this paper is recorded using a 14 channel Emotiv Epoc neuro-headset with a sampling rate of $128 \mathrm{~Hz}$ and an in-built band-pass filter of 0.2-45 Hz. The electrodes: AF3, F7, F3, FC5, T7, P7, O1, O2, P8, T8, FC6, F4, F8, and AF4, are arranged on the basis of the standard 10-20 system (Fig. 2) [38]. Nine healthy subjects with no prior experience on BMI (six male and three female, one left-handed and eight right-handed), participated in this experiment over a period of two days. In the first day, the subjects perform the tasks on two separate sessions. The data from the first session is used to train the decoder, while the same from the second session is used for offline testing the training of the decoder. In the second day, the subjects would control the movement of a robot arm in real-time based on the decoder trained on the previous day. Since, we are dealing with human subjects for experimental purpose, we abide by the norms of Helsinki Declaration of 1975, revised in 2000. Prior to the experiments, the subjects are informed about the purpose of the experiment and the tasks they have to perform.

2) Task and Stimuli: The experiment designed for this paper is divided into two phases: 1) offline and 2) online. In the offline phase, we determine the parameters of the support vector machines (SVMs) decoder for each subject. We perform an offline validation of the adaptivity of the decoder prior to employing it for the online phase.

The training and offline testing sessions comprise instructing the subjects through a sequence of visual stimulus to imagine the movement of the corresponding MI task, which is, left and right movement. Fig. 3 shows the generic structure of the visual cue. First, a blank screen is projected to the subject for $20 \mathrm{~s}$, which provides the baseline of the EEG. Then, a fixation " + " is displayed on screen for $1 \mathrm{~s}$ which is an indicator to the subject to get ready for the task. Next, the 


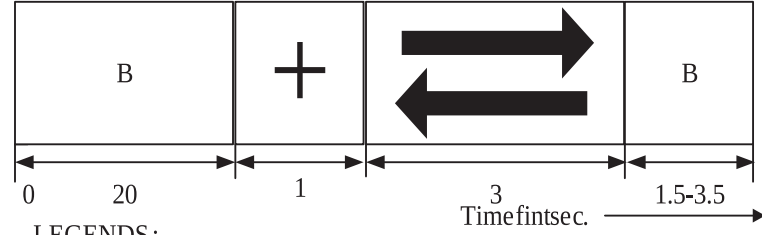

LEGENDS :

B-BlanktScreen ,t+t-tFixationtCross,

RighttImagery ,

LefttImagery

Fig. 3. Timing diagram of a single trial to train the subject in left and right hand MI (as indicated by left and right arrow, respectively).

instructions are provided to the subject for $3 \mathrm{~s}$ in form of arrows. According to the direction of the arrow, the subject imagines either left or right hand movement. Following the instructions, a blank screen is again displayed for 1.5-3.5 s. It allows the subject to relax during the task and removes the possibility of over-lapping between two mental states. Each task is repeated 40 times for the training session and 20 times for the offline testing session.

During the online tasks, the subjects are not shown any visual cues but are provided with audio cues from the operator. The operator would instruct the subject to move toward the top or bottom target (shown as black dots in Fig. 3). The subject would then generate the necessary MI commands to move the robot toward the target. The sequence of the instructions are random in nature and he/she would take several discrete steps (MI trials) to reach the target. The control commands required to move the robot is as follows: 1) left MI indicates upward movement of the robot and 2) right MI indicates downward movement of the robot. The subject observes the movement of the robot arm, which is considered as FB to the subject. If the decoder makes an error by producing the wrong output, then the subject on observing the error would attempt to fix it by generating the right brain signal.

The robot used in this experiment has 3-DOF and is located in Brain Science Institute-Toyota Collaboration Center, RIKEN, Japan. The decoder output commands from the decoder are sent remotely through an secure shell (SSH) file transfer protocol [39] from INRIA-LIRMM, France. Prior to the subject sending commands to the robot, peripheral motor controller is trained using synergetic learning to adapt to the given dynamical environment including arm inertial configuration and the newly given end-point load which influences interaction torques of multijoints in complex way. Here, the online task required the subject to guide the robot end-point toward the target based on the instructions from the operator. The online experimental task was repeated twice for each weight.

\section{Co-Adaptive EEG-BMI System}

The BMI system employs wavelet transforms [40], [41] for feature extraction, Laplacian EigenMaps [42] to determine the relevant features and an SVM classifier [43] to decode between the two mental states. The BMI system achieves co-adaptivity by the method mentioned in Section II-A.

1) Preprocessing: It is known from [4] and [38] that MI signals are characterized by the presence of event ERD/ERS [44], which are dominant in $\mu(8-12 \mathrm{~Hz})$ and central $\beta(16-24 \mathrm{~Hz})$ band of the EEG [38]. We preprocess the raw EEG data by applying a band-pass filter in $8-25 \mathrm{~Hz}$ range using a 4th order elliptical filter of $1 \mathrm{~dB}$ passband ripple and $30 \mathrm{~dB}$ stopband ripple [15]. Elliptical filters are characterized by a very sharp frequency roll-off and is equiripple in nature, which provides good attenuation of the pass- and the stop-band ripples [45]. With this step, noise due to muscle or eye movement, environmental interference and other parallel brain processes (not related to the tasks) is also removed.

2) Feature Extraction: The filtered signals are then processed using discrete wavelet transform (DWT) [41] to derive the signature features related to left- and right-MI. Wavelet transform provides localized frequency information over a given time period, which is highly suitable for nonstationary signals like EEG. The DWT decomposes the signal at different resolutions into coarse approximation and detail coefficient [41].

In this paper, we have selected Daubechies wavelet of the fourth order as the mother wavelet. As mentioned earlier, MI signals are dominant in the 8-12 Hz and 16-24 Hz range. We have extracted $3 \mathrm{~s}$ of EEG from onset of every stimuli, decomposed it to its fourth level and then reconstructed it using only the third and fourth detail coefficient. The final feature vector is constructed from the average of the reconstructed signal at D3 and D4 level. Thus, the final dimension of the feature vector for each trial is 384 features $\times 14$ electrodes.

3) Feature Selection: Sometimes due to high dimensionality of the features, the decoder suffers from high computational time, lack of relevant information, and overfitting, which in turn has a detrimental effect on the performance of the BMI. To negate this problem, researchers employ some form of linear or nonlinear dimensionality reduction technique [46], [47]. Laplacian EigenMap [42] is an unsupervised manifold learning algorithm which performs nonlinear dimensionality reduction by the following four basic steps.

1) Compute the nearest neighbors of the input data.

2) Using neighborhood relations construct a weight graph matrix.

3) Optimize the graph matrix based on a fitness function.

4) Project the final data from the top or bottom half of the matrix.

Extensive details on Laplacian EigenMaps are given in [42]. The advantage of this technique is to provide an optimal embedding solution to the manifold, for interpreting the dimensionality reduction problem geometrically, by maintaining the locality and proximity relations. Thus, it is insensitive to outliers and noise.

In this paper, we have determined the optimal dimensionality of relevant features for each subject from their validation results. The dimension which yields the best accuracy is used during online testing. The dimension of the reduced feature vector for each subject is mentioned in Table II.

4) Decoder Design: Selection of a classifier algorithm is also an important issue. SVM [43] nowadays has earned popularity for its good recognition accuracy and speed. Training time of SVM is significantly small in comparison to naive Bayesian and multilayered perceptron [43]. This motivated us to select SVM in the present application. 


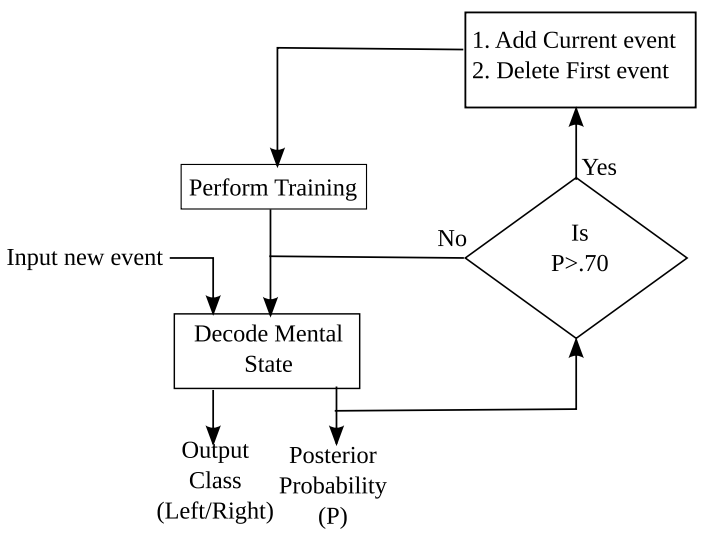

Fig. 4. BMI adaptive scheme employed in this paper.

An SVM classifier maps the input vectors in high dimensional feature space through some nonlinear mapping which can easily separate the data point vectors of two classes by a hyperplane. The hyperplane is a linear decision function constructed from a small amount of training data, called support vectors. The optimal hyperplane is a plane having the maximum distance from support vectors of the two classes. The data points placed beyond the margins of the hyperplane are easily classified into their respective classes. The aim of the SVM classifiers is to determine the separating hyperplane with the maximum margin.

To make the decoder adaptive to the changing brain state of the subject, we have employed the posterior probability $(P)$ of the data-points, which is described as follows.

1) Initially train the decoder (SVM in this paper) using $L(L>0.5 \times N)$ datapoints, where $N$ is the total number of datapoints.

2) Input $N-L$ datapoints to the trained decoder and determine their respective posterior probabilities $(P)$.

3) If $P>0.7$, include the current datapoint and remove the first datapoint to create a new feature vector.

4) Retrain the decoder and repeat from step 1.

To decide on the optimal value of $P$, we determined the accuracy of the classifier for different values of $P$ from $[0,1]$ with step-size of 0.1 . The value which provided the optimal result is selected as the final value for offline validation and online testing. The influence of $P$ with different value is discussed on the result section. A simplified block diagram of the steps involved in the adaptive process is illustrated in Fig. 4.

\section{Peripheric Motor Learning of the Dynamic Environment}

Tacit learning employs the command signal accumulated during repetitive interaction with the environment to develop an appropriate behavior for the system. The motor learning paradigm for multijoint reaching coordination, first proposed in [26] and shown in Fig. 5, can be summarized as follows.

1) The subject intends to move toward the target, which is represented by the direction to the target, and it is

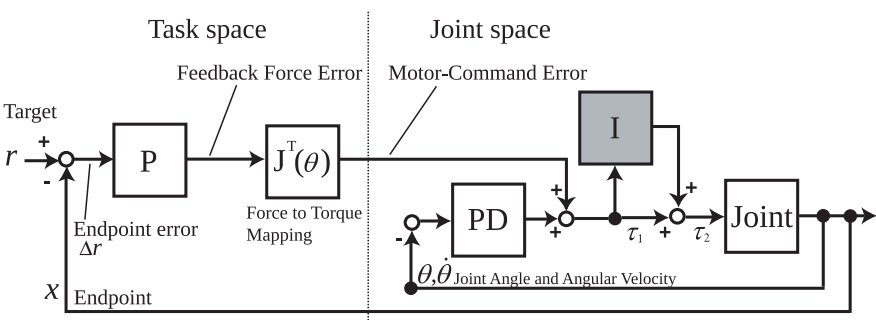

Fig. 5. Block diagram of the tacit learning-based synergetic motor control paradigm.

regarded as the proportional $(P) \mathrm{FB}$ error between the target and current endpoint.

2) The FB force error is mapped into the joint torque space by using the Jacobian of the robot arm and the motorcommand error works as a supervising signal.

3) Here, the local proportional derivative (PD) control mainly relates to a local reflex loop which regulates the joint angles to change smoothly.

4) The torque command accumulation part, defined by an integral $I$, serves as a unique learning process which corresponds to tacit learning. This portion learns to compensate the interaction torques such as gravitational and limb inertia components, and converts into a predictive torque pattern following the motor learning.

Specifically, the controllers for tacit learning can be expressed as follows:

$$
\begin{aligned}
\boldsymbol{\tau}_{1}= & -\boldsymbol{J}^{T}(\boldsymbol{\theta}) k \Delta \boldsymbol{r}-\boldsymbol{A} \Delta \boldsymbol{\theta}-\boldsymbol{B} \dot{\boldsymbol{\theta}} \\
\boldsymbol{\tau}_{2}= & -\boldsymbol{J}^{T}(\boldsymbol{\theta}) k \Delta \boldsymbol{r}-\boldsymbol{A} \Delta \boldsymbol{\theta}-\boldsymbol{B} \dot{\boldsymbol{\theta}}+\boldsymbol{C} \int \boldsymbol{\tau}_{1} d t \\
& \boldsymbol{\tau}_{1}, \boldsymbol{\tau}_{2}, \Delta \boldsymbol{\theta}, \dot{\boldsymbol{\theta}} \in R^{m}, \Delta \boldsymbol{r} \in R^{n}, \boldsymbol{J}^{T}(\boldsymbol{\theta}) \in R^{m \times n}
\end{aligned}
$$

where, $m$ is the number of joints, $n$ is the dimensional number of the task space, $\boldsymbol{\tau}_{\mathbf{2}}$ implies the control torque inputs of the joints during tacit learning. $\boldsymbol{\theta}$ and $\dot{\boldsymbol{\theta}}$ refers to the angles and angular velocities of joints, respectively. $\boldsymbol{J}^{T}(\boldsymbol{\theta}) k \Delta \boldsymbol{r}$ corresponds to the neural substrate of force mapping functionality presumably due to corticospinal control [48]. $\boldsymbol{A}$ and $\boldsymbol{B}$ are diagonal matrices which contain the proportional and derivative gains of the PD controller and $\boldsymbol{C}$ is made of the gains of the torque command integration regarding motor-command error and local FB torque. The PD controller corresponds to a local reflex loop as a function of muscle spindles. Even though this paper is a joint-level representation, the resisting features against muscle length change and velocity change can still be captured by the resisting feature in the joint angle and angular velocity changes as in local PD controller. The robot consists of the upper arm, forearm and hand segments with a 3-DOF configuration. Each joint is actuated using a DC motor with an encoder and a harmonic drive gearing for backdrivability. Each motor is current-controlled with servo-amplifier drives. Thus, each joint has a local torque control to generate the specified joint torque for the robot. The control algorithms are executed on a master PC with the interface of analog-todigital and digital-to-analog converters from the encoders and to the motors, respectively. This manipulator is redundant as the three motor axes are in parallel. 
The detailed performance of the synergetic learning controller is mentioned in [26]. The configuration allows the joints to be controlled independently and thus it can be presumed as a modular structure present within cerebellar pathways. Typical optimal solutions are based on model-based cost approach, but this approach optimizes the process by repetitive interaction with the environment rather than using some cost function. This learning controller turns into predictive control from initial FB control while synergetically modifying the redundant joint space usage toward energy efficient solutions. The joint synergy may appear through the environmental interactions.

\section{E. BMI Evaluation Metrics}

To evaluate the performance of the BMI system during training and validation, we have employed four quantitative measures. They are: 1) classification accuracy [49]; 2) sensitivity [49]; 3) specificity [49]; and 4) area under the curve (AUC) [50].

Now, the definition of the above evaluation parameters, where TP is the true positive, TN is the true negative, FP is the false positive, and FN is the false negative, is as follows.

1) Classification Accuracy: It is the measure of how correctly a classifier can predict a class. It is given by

$$
\text { Accuracy }=\frac{\mathrm{TP}+\mathrm{TN}}{\mathrm{TP}+\mathrm{TN}+\mathrm{FP}+\mathrm{FN}} \text {. }
$$

2) Sensitivity: It is the measure of how correctly a classifier has classified the positive class. It is given by

$$
\text { Sensitivity }=\frac{\mathrm{TP}}{\mathrm{TP}+\mathrm{FN}} \text {. }
$$

3) Specificity: It is the measure of how correctly a classifier has classified the negative class. It is given by

$$
\text { Specificity }=\frac{\mathrm{TN}}{\mathrm{TN}+\mathrm{FP}} \text {. }
$$

4) Area Under the Curve: AUC is derived from the receiver operating characteristic (ROC) [51] curve of the classifier performance. ROC is a curve between true positive rate (sensitivity) in the $y$-axis and false positive rate (1-specificity) in the $x$-axis obtained by varying the decision boundary. Perfect classification is denoted by a point in the upper left corner $(0,1)$ indicating $100 \%$ specificity and $100 \%$ sensitivity. The random guess line is the line joining $(0,0)$ and $(1,1)$ and contains the point $(0.5,0.5)$. Points in the upper portion of the random guess line indicate good prediction and the points below the line indicate poor prediction. Thus, we can say ROC curve is a plot of the classification result of the most positive classification to the most negative classification and the resultant AUC is widely used as a classification metric.

We have quantified the performance of the online task of moving the robot arm using left and right hand MI by the following metrics: 1) accuracy and 2) time taken, i.e., the time taken to process and decode the incoming EEG signal and transmit it remotely to the robot using SSH protocol.
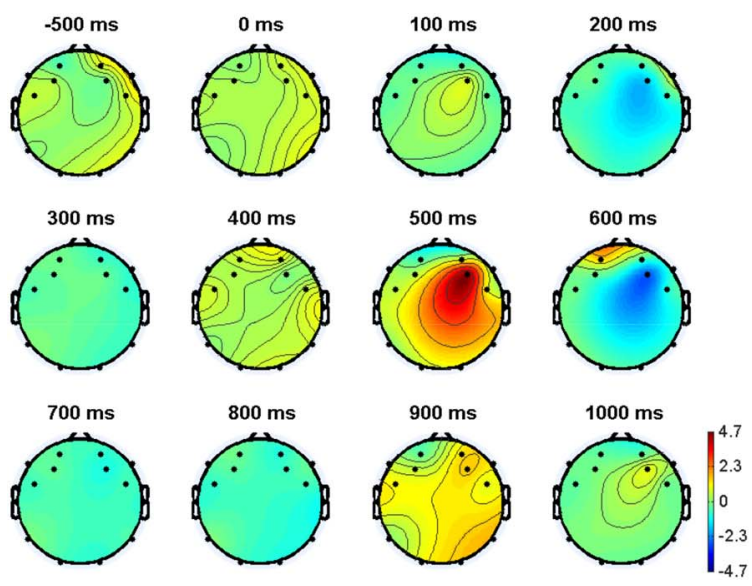

(a)
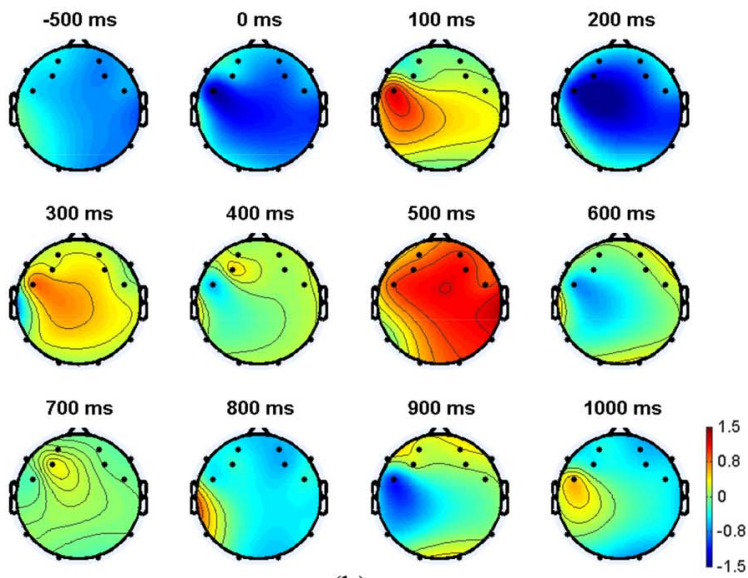

(b)

Fig. 6. Topographical plot of brain activation during -500 to $1000 \mathrm{~ms}$ for (a) left-hand MI and (b) right-hand MI for subject 6.0 ms marks the onset of the task.

\section{RESULTS}

This section begins with the detection of ERD/ERS signals from the EEG acquired from the Emotiv system. Then, it presents the results on the performance of the BMI system during training and offline testing of the decoder, performance of the peripheral motor controller during its learning stage and the complete performance of BMI system (which includes the trained BMI decoder and the trained peripheral motor controller) during online experimentation. The offline processing and online experimentation has been executed in MATLAB Windows 8.1 environment.

\section{A. Detection of ERD/ERS Patterns}

The Emotiv acquisition system, used in this paper, does not have any channels directly over the primary motor cortex, but it has channels, FC5, FC6, P7, and P8, in the vicinity of the region. Thus, for MI studies, one can use these channels to detect the ERD/ERS waveform. Emotiv is a low-cost, portable EEG acquisition system and thus, in recent years, researchers have used it in detection of P300, steady state visually evoked potential, and ERD/ERS waveform. Hurtado-Rincon et al. [52] and Dharmasena et al. [53] has successfully classified between 
TABLE I

Training Results of the Decoder During No Adaptation And Adaptation

\begin{tabular}{|c|c|c|c|c|c|c|c|c|c|}
\hline Subjects & \multicolumn{4}{|c|}{ No Adaptation } & \multicolumn{4}{c|}{ Adaptation } & p-value \\
\hline & Accuracy & Sensitivity & Specificity & AUC & Accuracy & Sensitivity & Specificity & AUC & \\
\hline S1 & 88.75 & 90.91 & 88.41 & 79.30 & 93.75 & 92.68 & 94.87 & 93.50 & 0.005 \\
S2 & 90.00 & 91.30 & 88.24 & 95.65 & 97.50 & 95.24 & 100.00 & 100.00 & 0.001 \\
S3 & 68.75 & 69.23 & 68.29 & 76.81 & 78.75 & 79.73 & 66.67 & 71.27 & 0.0002 \\
S4 & 75.00 & 73.81 & 76.32 & 78.5 & 92.50 & 92.86 & 92.11 & 93.67 & 0.001 \\
S5 & 68.75 & 70.27 & 67.44 & 76.25 & 83.75 & 81.82 & 86.11 & 85.37 & 0.006 \\
S6 & 77.50 & 77.50 & 77.50 & 82.63 & 90.00 & 93.81 & 86.11 & 90.22 & 0.03 \\
S7 & 75.00 & 77.93 & 83.10 & 77.93 & 80.00 & 78.57 & 81.58 & 82.06 & 0.055 \\
S8 & 77.50 & 77.50 & 77.50 & 82.38 & 80.00 & 80.00 & 80.00 & 87.63 & 0.045 \\
S9 & 86.25 & 88.89 & 76.47 & 91.83 & 92.50 & 90.48 & 94.74 & 94.88 & 0.03 \\
\hline Mean & 78.61 & 79.70 & 78.14 & 82.36 & 87.64 & 87.24 & 86.91 & 88.73 & \\
SD & 8.01 & 8.59 & 7.50 & 6.88 & 7.05 & 7.00 & 10.06 & 8.48 & \\
\hline
\end{tabular}

TABLE II

Validation of the Decoder on a New Dataset (of 40 Trials) During No Adaptation and Adaptation

\begin{tabular}{|c|c|c|c|c|c|c|c|c|c|c|c|}
\hline Subjects & \multicolumn{5}{|c|}{ No Adaptation } & \multicolumn{5}{|c|}{ Adaptation } & p-value \\
\hline & Accuracy & Sensitivity & Specificity & AUC & FS & Accuracy & Sensitivity & Specificity & AUC & FS & \\
\hline S1 & 75.00 & 70.00 & 80.00 & 65.00 & 96 & 90.00 & 80.00 & 100.00 & 90.00 & 32 & 0.0065 \\
\hline S2 & 80.00 & 60.00 & 100.00 & 84.00 & 46 & 90.00 & 80.00 & 100.00 & 90.00 & 24 & 0.002 \\
\hline $\mathbf{S 3}$ & 75.00 & 60.00 & 90.00 & 68.00 & 22 & 75.00 & 90.00 & 60.00 & 68.00 & 10 & 0.07 \\
\hline S4 & 80.00 & 80.00 & 80.00 & 82.00 & 22 & 75.00 & 80.00 & 79.00 & 79.50 & 89 & 0.105 \\
\hline S5 & 70.00 & 70.00 & 70.00 & 67.00 & 19 & 80.00 & 80.00 & 80.00 & 70.00 & 34 & 0.005 \\
\hline S6 & 70.00 & 70.00 & 70.00 & 64.00 & 25 & 85.00 & 100.00 & 70.00 & 80.00 & 46 & 0.002 \\
\hline S7 & 75.00 & 80.00 & 70.00 & 75.00 & 27 & 75.00 & 80.00 & 70.00 & 74.00 & 27 & 0.110 \\
\hline S8 & 75.00 & 90.00 & 60.00 & 68.00 & 39 & 85.00 & 90.00 & 80.00 & 81.00 & 29 & 0.005 \\
\hline S9 & 80.00 & 70.00 & 90.00 & 82.00 & 88 & 90.00 & 90.00 & 90.00 & 83.00 & 21 & 0.001 \\
\hline Mean & 75.56 & 72.22 & 78.89 & 72.78 & & 82.78 & 85.56 & 81.00 & 79.50 & & \\
\hline SD & 3.91 & 9.72 & 12.69 & 8.04 & & 6.67 & 7.26 & 13.66 & 7.81 & & \\
\hline
\end{tabular}

left- and right-MI. Fok et al. [54] have acquired brain signals related to movement to successfully drive a powered orthosis tasked at opening and closing of the patient's hand.

Fig. 6 provides a topographical plot of brain activation during -500 to $1000 \mathrm{~ms}$ for left- and right-hand MI tasks for subject 6 . As noted from the plots, the right side of the brain is more active during left hand MI and vice-versa. Also, the frontoparietal region of the brain is more active than other regions. The inferences derived from the plots confirms the presence of ERD/ERS.

\section{B. BMI Adaptive Decoder Training and Validation}

To analyze the training performance of the decoder, we have used $k$-fold cross validation technique [49], where we have taken the value of $k$ to be 7 to lower the variance of the outcome. In Table I, we have shown the average of the classification metric [i.e., accuracy, sensitivity, specificity, and AUC (in \%)] for all the nine subjects. In the same table, we have also compared the results of the decoder while it is adapting (adaptation column in the table) and when we did not include the adaptation to the decoder (no adaptation column in the table). A significant increase in the performance metric is noted for all the subjects when we have included our adaptation scheme to the BMI. The adaptation result suggests an increase of average accuracy, sensitivity, specificity, and AUC by $9.03 \%, 7.54 \%, 8.77 \%$, and $6.37 \%$, respectively, from its nonadaptive counterpart. We further statistically validate the results of the nonadaptive and adaptive decoder using a 5by-2 paired $t$-test [55], where the null hypothesis states that the nonadaptive decoder is at most as accurate as the adaptive decoder. The $p$-values as observed from Table I suggests that for all subjects rejects the null hypothesis at 5\% significance level, and thus, it is statistically shown that the adaptive decoder is more accurate than the nonadaptive one.

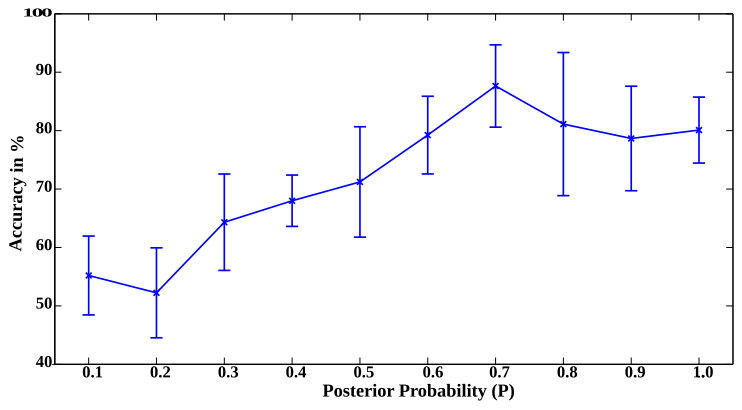

Fig. 7. Average accuracies obtained over nine subjects, while changing the limiting posterior probability from 0.1 to 1.0 .

The average accuracy indicates that the decoder can detect $87 \%$ of the incoming features to be true. Sometimes, the accuracy may provide a skewed result by detecting one class very well but the other class very poorly. Thus, the sensitivity, specificity and AUC provides a more reliable result in this context. Sensitivity suggests how good the decoder is to detect a positive class, while sensitivity suggests how good it detects the negative result. The AUC on the other hand, indicates the tradeoff between the sensitivity and specificity to make the decoder produce an optimal result. The average sensitivity, specificity and AUC being more than $85 \%$ suggests that the decoder can detect $85 \%$ of the positive and negative classes without adversely affecting each other.

Through Fig. 7, we also show the influence of changing the posterior probability $(P)$ on the decoder output. On changing $P$ from 0.1 to 1.0 , there is a gradual increase of the average accuracies (of the training data) till $P>0.7$ and then it decreases. From this observation, we have selected $P>0.7$.

Next, we validate the performance of our BMI decoder over a new independent dataset, which for this paper is the second 


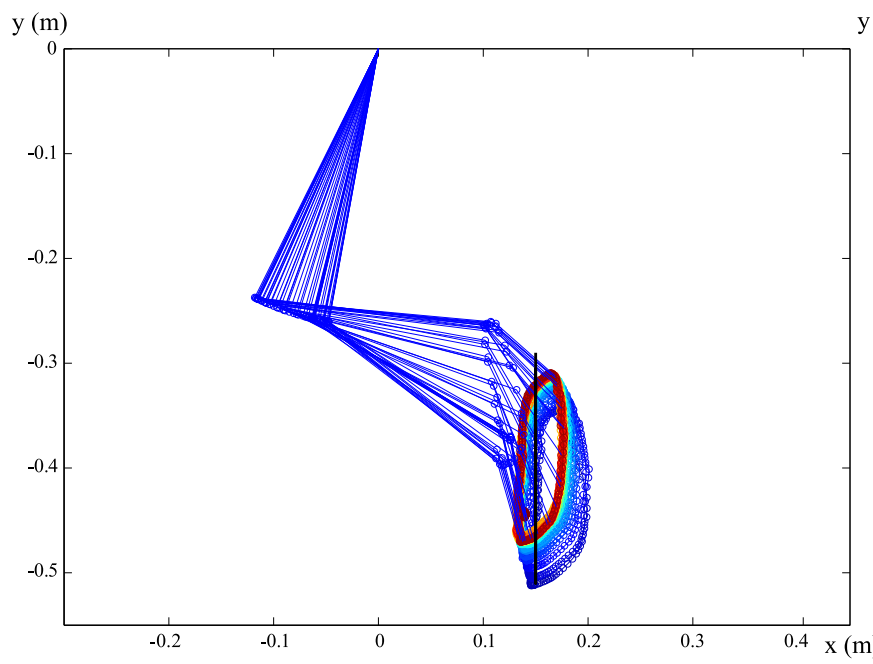

(a)

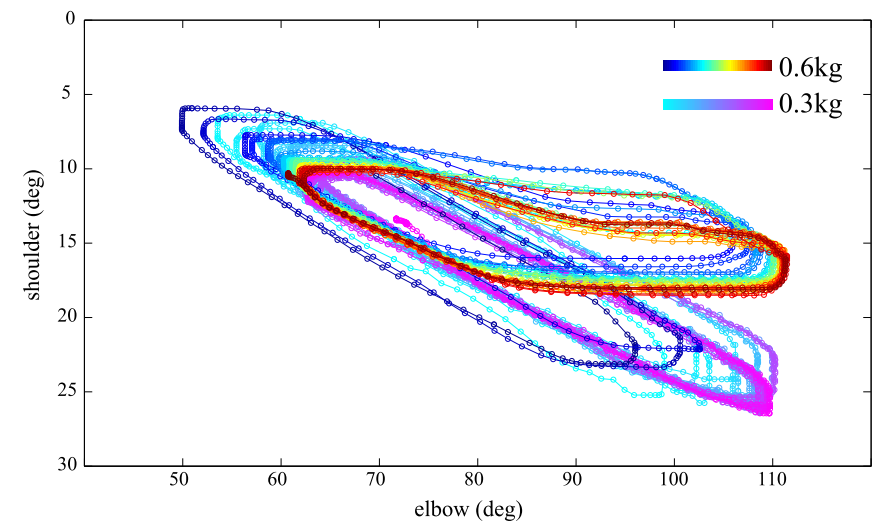

(c)

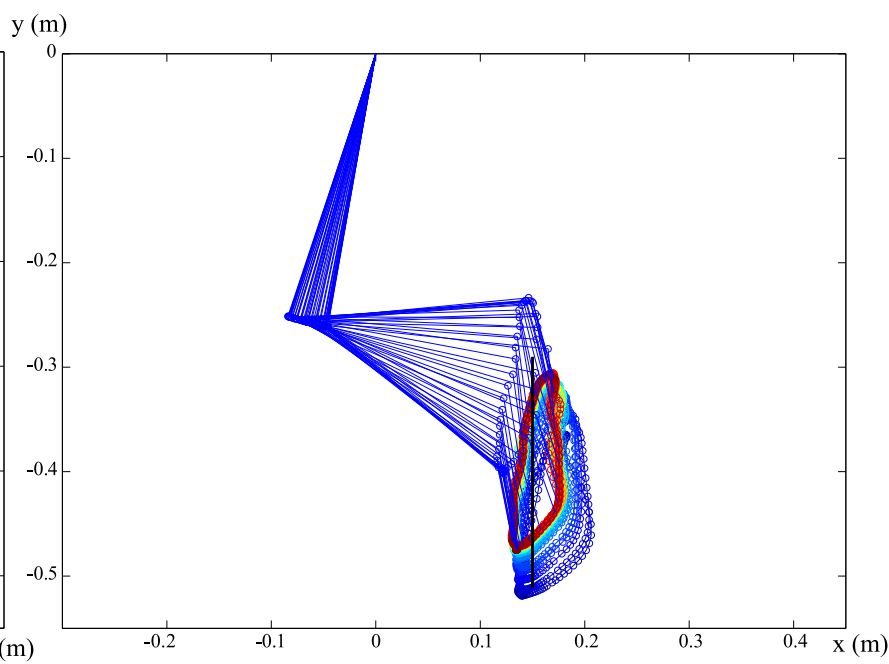

(b)

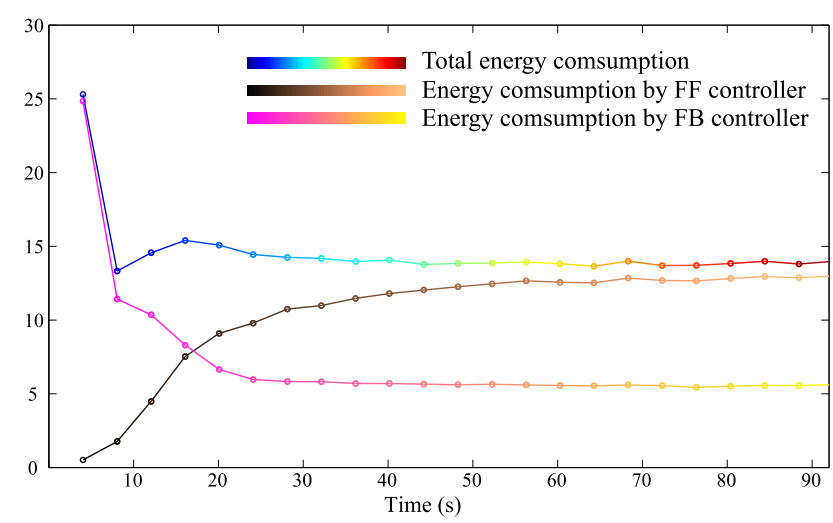

(d)

Fig. 8. Endpoint transition of robot with load (a) $300 \mathrm{~g}$ and trajectory of the shoulder-elbow-wrist joints for $300 \mathrm{~g}$ load and (b) $600 \mathrm{~g}$ and trajectory of the shoulder-elbow-wrist joints for $600 \mathrm{~g}$ load. (c) Phase potrait of joint angle-angular velocity for the shoulder-elbow joints at different weight conditions. (d) Energy consumption of time sequential changes in one cycle of up/down motion with $600 \mathrm{~g}$ load along with its FB component and FF component.

offline session. This step is included in this paper to check the performance of the classifier on a new dataset, prior to using it for online experimentation and confirm its ability to perform at par with its training result. Here, the features of the independent dataset is fed directly to the trained classifier, which provides the output. The results of validating the decoder is presented in Table II. We notice a slight decrease in accuracy, sensitivity, specificity and AUC by $4.86 \%, 1.68 \%$, $5.91 \%$, and $9.23 \%$ from its training counterpart. The slight decrease can be attributed to the nonstationarity of the EEG and due to some fatigue encountered by the subjects after the training session. Anyways, the decrease is not large and the decoder can detect the required class correctly by $75 \%$ for all the subjects where for subject 1, 3, and 9, it can detect $90 \%$ of the signals correctly. The result also suggests that the decoder did not overfit during training. Similar to the training results, on inclusion of the adaptive paradigm, an increase of average accuracy, sensitivity, specificity, and AUC is noted by $7.22 \%$, $13.34 \%, 2.11 \%$, and $6.72 \%$, respectively, from its nonadaptive counterpart. Again we statistically validate the adaptive decoder with its nonadaptive counterpart using 5-by-2 paired $t$-test for the same null hypothesis. As noted from the $p$-values of Table II, seven of the nine subjects rejects the null hypothesis at 5\% significance level and thus for these subjects the adaptive decoder are more accurate than its nonadaptive counterpart. But for subjects 4 and 7, the null hypothesis is accepted and thus the adaptive decoder is as accurate as its nonadaptive counterpart.

From the validation dataset, we also determine the best dimension of features for each subject which is shown in the FS column of the table. It is noted from the table that the value of FS not only changes for each subject but also during no adaptation and adaptation circumstances. It shows how each subject varies from each other and as a result each subject has his/her own individual decoder trained. The positive result shown during validation allowed us to use the decoder during online testing of the BMI system.

\section{Learning of the Synergetic Motor Controller}

For this experiment, we have used two different loads of 300 and $600 \mathrm{~g}$ as unknown loads for the robot. It means the load 


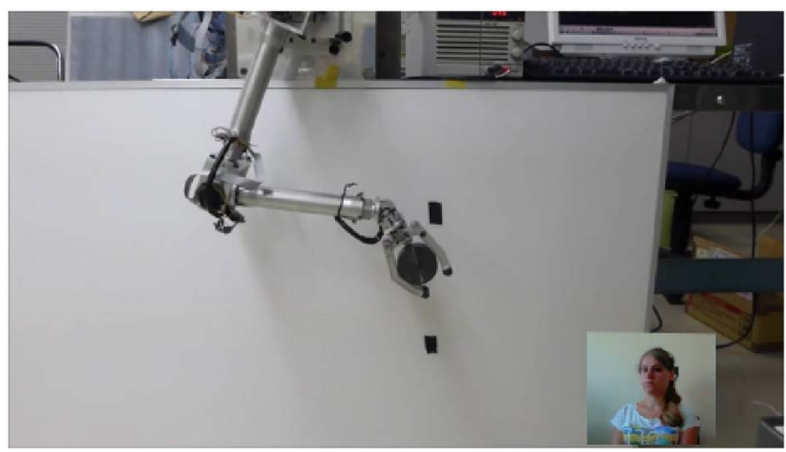

Fig. 9. Subject performing the online control of multi-DOF robot in a simultaneous way by using co-adaptive BMI. The black dots indicate the targets for the subjects in the vertical plane.

is not a-priori known for the motor controller. For both the weights, prior to the subject sending commands to the robot, peripheral motor controller is trained using our approach for $70 \mathrm{~s}$ to adapt to the given dynamical environment including arm inertial configuration and end-point load.

Fig. 8 describes the learning of the peripheral motor controller using tacit learning. Fig. 8(a) and (b) illustrates the trajectory of the robot arm during its learning for both the loads. The time sequential transition of the end-point of the robot for both the figures are illustrated using a color map which changes with the progress of time. The lines (in blue) show the trajectory of each individual links. The black bar represents the target limit. Both the figures demonstrate the ability of the synergetic controller to adapt the multijoint usage in redundant space to the different load conditions by changing the motion range of each angle joints.

Fig. 8(c) shows the shoulder-elbow phase map for the different weights. The figure illustrates that the shoulder is used less when the load gets heavy, while the wrist usage is also minimized to reduce the total energy consumption for the same task.

Fig. 8(d) presents the temporal transition of energy consumption in synergetic learning control with a $600 \mathrm{~g}$ load. The energy consumption was calculated by summing up each joint energy consumption by integrating $\tau \dot{\theta}$, and dividing it by time to compute as work rate (power). From (4), let us consider the torque component of PD FB as an FB controller and the integral term as a feedforward (FF) controller. During learning, the contribution of FF is increased until the torque from FF converges into a certain pattern. Thus, synergetic learning matches with the typical human motor learning process. As noted from the figure, motion is initially generated by endpoint error principally (influence of $\mathrm{FB}$ ), but this part is being minimized and predictive FF pattern takes over during the learning of the lifting up/down motion.

\section{Online Performance of the Simultaneous Multi-DOF Robot Control by Co-Adaptive BMI}

Following the training of robot controller using synergetic motor learning algorithm, the subject is ready to move the robot arm by his/her motor intention. An example of the subject performing the task is shown in Fig. 9. The subject from

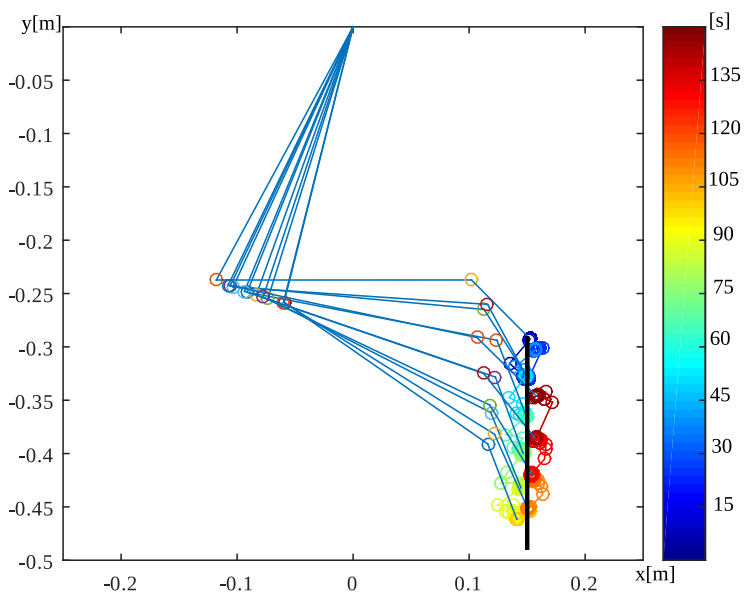

(a)

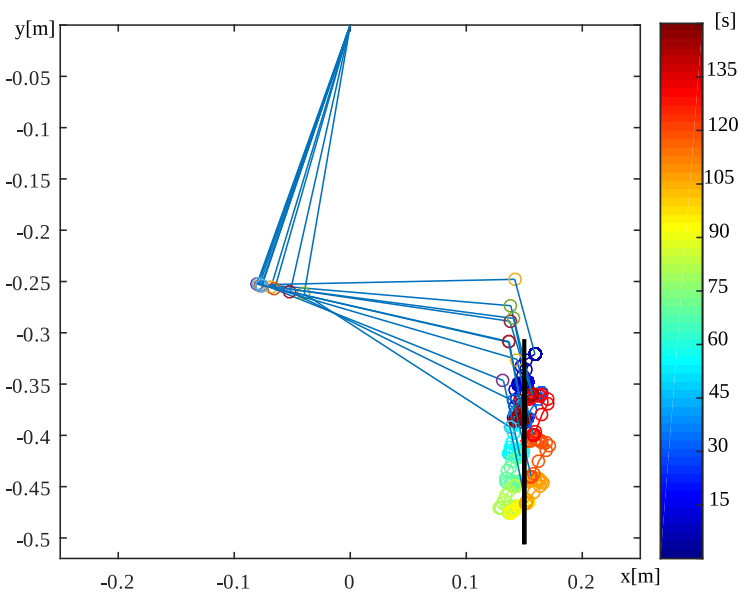

(b)

Fig. 10. Trace of the movement of the robot arm during simultaneous multiDOF robot control which is driven by the subject motor intention through co-adaptive BMI. The robot is holding (a) $300 \mathrm{~g}$ load, (b) $600 \mathrm{~g}$ load. The joint angle variance on shoulder and wrist for the heavier weight condition has become half compared to lighter weight condition.

one end generates the instructed brain signals (left or right MI). The decoder decodes the brain signal to generate the corresponding control command necessary to move the robot in either up or down direction. The command is transferred to the robot via SSH remote communication. The online task requires the subject to move the robot between the two targets (shown as black dots in Fig. 9).

Fig. 10 shows an example of the trace of the movement of the robot arm [similar to Fig. 8(a) and (b)] which is driven by the subject motor intention through co-adaptive BMI. As seen from the figure, the robot requires a number of steps (or trials of MI extraction) to reach the target. The time sequential transition of the end-point of the robot for both the figures are illustrated using a color map which changes with the progress of time. As noted from the figures, when the robot carries a heavier weight (i.e., $600 \mathrm{~g}$ in this paper), there is a smaller displacement in the shoulder and wrist joint as compared to a lighter weight condition (i.e., $300 \mathrm{~g}$ in this paper). This observation is also validated by the joint angle variance metric shown in Table III. The results from the table that the joint angle variance on shoulder $\left(1.67^{\circ}\right)$ and wrist $\left(2.03^{\circ}\right)$ for the 
TABLE III

Online Performance on Multijoint Robot Arm Adaptive BMi Control With Different Load Conditions

\begin{tabular}{|c|c|c|c|c|c|c|c|c|c|c|}
\hline Load & \multicolumn{5}{|c|}{$300 \mathrm{~g}$} & \multicolumn{5}{|c|}{$600 \mathrm{~g}$} \\
\hline & & & \multicolumn{3}{|c|}{$\begin{array}{l}\text { Joint Angle Variances } \\
\text { in degree }\end{array}$} & & & \multicolumn{3}{|c|}{$\begin{array}{c}\text { Joint Angle Variances } \\
\text { in degree }\end{array}$} \\
\hline & Accuracy & total time & Shoulder & Elbow & Wrist & Accuracy & total time & Shoulder & Elbow & Wrist \\
\hline S1 & 86.20 & 8.284 & 5.90 & 5.50 & 4.01 & 81.20 & 8.148 & 1.66 & 5.60 & 1.90 \\
\hline S2 & 75.30 & 8.752 & 6.07 & 4.80 & 4.35 & 73.70 & 8.675 & 2.01 & 5.06 & 1.65 \\
\hline S3 & 72.20 & 9.671 & 5.56 & 5.25 & 5.01 & 76.38 & 9.526 & 2.18 & 6.48 & 1.50 \\
\hline S4 & 65.55 & 7.719 & 5.39 & 5.78 & 5.21 & 74.85 & 9.166 & 1.55 & 5.87 & 2.12 \\
\hline S5 & 76.75 & 8.515 & 5.33 & 5.41 & 5.20 & 83.65 & 8.812 & 1.43 & 6.20 & 2.61 \\
\hline S6 & 80.10 & 8.876 & 5.67 & 5.80 & 5.80 & 74.45 & 9.194 & 1.26 & 6.00 & 2.64 \\
\hline S7 & 74.95 & 7.599 & 5.58 & 5.24 & 5.90 & 80.70 & 8.213 & 1.49 & 5.13 & 1.49 \\
\hline S8 & 76.15 & 8.447 & 5.33 & 5.92 & 5.90 & 78.50 & 8.334 & 1.43 & 5.91 & 2.53 \\
\hline S9 & 84.30 & 8.542 & 6.13 & 5.87 & 5.00 & 78.60 & 8.208 & 2.06 & 5.96 & 2.04 \\
\hline Mean & 76.83 & 8.489 & 5.66 & 5.51 & 5.15 & 78.004 & 8.697 & 1.67 & 5.80 & 2.03 \\
\hline SD & 6.21 & 0.617 & 0.306 & 0.372 & 0.664 & 3.42 & 0.509 & 0.327 & 0.466 & 0.494 \\
\hline
\end{tabular}

heavier weight condition has become less than half compared to lighter weight condition $\left(5.66^{\circ}\right.$ for shoulder and $5.15^{\circ}$ for wrist). This joint usage variation appears automatically without changing any setting in the controller. By using prioceptive information such as torque and joint angles, this adaptation systematically appears through the synergetic learning control [26]. This observation regarding minimal shoulder and wrist usage for heavy object manipulation is well matched to the situation in human motor control. Holding heavy object in the air requires redundant torque production if you hold it by using multiple joints. Thus, when we need to lift up the heavy object, it is efficient if we minimize the multiple joint usage to reduce total energy consumption.

Further, from Table III, the average accuracies of all subjects are over $75 \%$ with subject 1 producing an accuracy of $80 \%$ for both weights. The time taken to transfer each command over a remote connection to the robot is around $8.5 \mathrm{~s}$ which is considered to be a high value for real-time applications. Such high value may be attributed to the high processing time required by MATLAB and the time to transfer the commands from one computer to another through the Internet across different continents. The link to a video showing a subject controlling the multijoint robot is given in http://www.lirmm.fr/ hayashibe/SMC/ SynergeticBMIx2.5speed.mp4.

\section{Discussion}

In this section, we describe some co-adaptive approaches implemented by other researchers. A detailed comparison is difficult as the studies are different in terms of the paradigms and the performance evaluation. An intuitive comparison of the training and validation results of this paper is thus given to fit our results with existing literature.

Faller et al. [56] developed co-adaptive cue-based training paradigms for ERD-based BCI to benefit severely disabled patients, including ones with spinal cord injury. The $\mathrm{BCI}$ analyzes the EEG from three bipolar locations: 1) $\mathrm{C} 3$; 2) $\mathrm{Cz}$; and 3) $\mathrm{C} 4$ while the user performs left and right hand $\mathrm{MI}$ and relax with eye open tasks alternatively. The BCI auto-calibrates and provides FB about the MI tasks after 5 min of data collection. The BCI first performs outlier rejection and then selects the most discriminable logarithmic band-power (from 9 to 14 and 16 to $26 \mathrm{~Hz}$ ) as features for the linear disciminant analysis classifier. The mean average accuracy thus obtained for six tetraplegic participants $69.5 \% \pm 6.4$. In another similar work of Faller et al. [22], they developed a co-adaptive system which implements a noncontrol state which allows the system to be self-paced in nature. This system worked significantly better than chance for 18 of 22 users in 24 min of training and 11 of 22 users for the self-pace paradigm.

In another interesting work, Kus et al. [57] developed a BCI system which followed an asynchronous mode of operation, automatic selection of parameters based on initial calibration and incremental update of the classifier parameters from FB. In their study, the EEG was spatially filtered based on spectral power estimation and relevant features were selected based on mutual information criterion. The final feature vector are used to recognize the MI by using a multinomial logistic regression classifier. The participants performed right hand, left hand and foot MI based on instructions from a visual cue with an accuracy of $74.84 \%$.

As noted from the few studies discussed, most researchers adapt the BCI system either through training or by continuous update of the classifier. In this paper, the subject adapts to the instructions by visually observing the movement of the robot arm in real-time and space. We have employed this form of adaptation to the subject to make the task more realistic and practical. The system has two adaptive functions. First, the BCI system continuously adapts to the current mental state of the subject by providing higher weights to the most recent signals and lesser weights to the older signals. The second system side adaptation occurs in the peripheral motor controller which adapts to the dynamic environment the robot is exposed to. The advantage of a separate motor learning control scheme, even for 3-DOF joint control, allows the subject to focus on the lower dimensional endpoint control of the robot while the proprioceptive information from the robot is processed inside the peripheral motor control and adapts accordingly while performing simultaneous multijoint control.

The development of a tridirectional (user-decoder-robot) BCI control of multi-DOF control is a novel attempt toward its aim. The results obtained in this preliminary study demonstrate that the use of automated interfaces to solve redundancy of joint movement is indeed possible and the positive results encourage us to further dwell into developing a tridirectional adaptive system for rehabilitative and assistive systems [58], [59]. 


\section{CONCLUSION}

In this paper, we have proposed a new BMI paradigm which integrates an MI EEG to extract the target intention with adaptive decoder for cortical signals and a synergetic motor learning control to cope with the peripheral control of a multijoint redundant robot arm with environmental dynamics adaptation capability. The proposed method allowed for BMIcontrolled robot to employ different joint usage depending on the given payload systematically through the learning process. This paradigm takes into account the tridirectional adaptations between the user, the BMI system and the multi-DOF arm. To the best of the authors' knowledge, it is a first system which incorporates dual adaptive nature in each cortical level and peripheral motor control level in BMI. We also should note the fact that the robot is controlled with torque control manner and not with position control, which is highly recommended for human-machine interaction, and known as natural human control nature. The positive result, thus obtained, has opened a door to proceed forward in this research, but it was verified with simple task as a starting point.

Future direction in this line of research would involve the incorporation of including a quantitative measure of the visual FB and further refinement of the adaptive and peripheral learning algorithm. To improve the speed and robustness of the BCI control alogrithm, we would design a self-paced experiment with the provision of an error FB through EEG [15]. Also, we will increase the types and number of different environment conditions toward a unified BMI framework for multijoint control interface.

\section{REFERENCES}

[1] R. P. N. Rao and R. Scherer, "Brain-computer interfacing [in the spotlight]," IEEE Signal Process. Mag., vol. 27, no. 4, pp. 150-152, Jul. 2010.

[2] D. J. McFarland and J. R. Wolpaw, "Brain-computer interface operation of robotic and prosthetic devices," Computer, vol. 41, no. 10, pp. 52-56, Oct. 2008.

[3] J. J. Daly and J. R. Wolpaw, "Brain-computer interfaces in neurological rehabilitation," Lancet Neurol., vol. 7, no. 11, pp. 1032-1043, 2008. [Online]. Available: http://www.sciencedirect.com/ science/article/pii/S1474442208702230

[4] G. Dornhege, Toward Brain-Computer Interfacing (A Bradford book). Cambridge, MA, USA: MIT Press, 2007.

[5] J. R. Wolpaw, N. Birbaumer, D. J. McFarland, G. Pfurtscheller, and T. M. Vaughan, "Brain-computer interfaces for communication and control," Clin. Neurophysiol., vol. 113, no. 6, pp. 767-791, 2002. [Online]. Available: http://www.sciencedirect.com/ science/article/pii/S1388245702000573

[6] E. A. Martinez-Garcia, E. Gallegos, and K. S. Jaichandar, "Telepresence by deploying an avatar robot with brain-robot interfacing," in Proc. 7th IEEE Conf. Ind. Electron. Appl. (ICIEA), Singapore, Jul. 2012, pp. $144-149$.

[7] I. S. Kotchetkov, B. Y. Hwang, G. Appelboom, C. P. Kellner, and E. S. Connolly, Jr., "Brain-computer interfaces: Military, neurosurgical, and ethical perspective," Neurosurg. Focus, vol. 28, no. 5, p. E25, 2010.

[8] R. Scherer et al., "Toward self-paced brain-computer communication: Navigation through virtual worlds," IEEE Trans. Biomed. Eng., vol. 55, no. 2, pp. 675-682, Feb. 2008.

[9] N. E. Bunderson, "Real-time control of an interactive impulsive virtual prosthesis," IEEE Trans. Neural Syst. Rehabil. Eng., vol. 22, no. 2, pp. 363-370, Mar. 2014.

[10] M. van Vliet et al., "Designing a brain-computer interface controlled video-game using consumer grade EEG hardware," in Proc. ISSNIP Conf. Biosignals Biorobot. (BRC), Manaus, Brazil, Jan. 2012, pp. 1-6.
[11] D. Marshall, D. Coyle, S. Wilson, and M. Callaghan, "Games, gameplay, and BCI: The state of the art," IEEE Trans. Comput. Intell. AI in Games, vol. 5, no. 2, pp. 82-99, Jun. 2013.

[12] L. F. Nicolas-Alonso and J. Gomez-Gil, "Brain computer interfaces, a review," Sensors, vol. 12, no. 2, p. 1211-1279, 2012.

[13] J. D. Millan et al., "Combining brain-computer interfaces and assistive technologies: State-of-the-art and challenges," Front. Neurosci., vol. 4 , p. 161 , Sep. 2010.

[14] S. Bhattacharyya, A. Konar, and D. N. Tibarewala, "A differential evolution based energy trajectory planner for artificial limb control using motor imagery EEG signal," Biomed. Signal Process. Control, vol. 11, pp. 107-113, May 2014. [Online]. Available: http://www.sciencedirect.com/science/article/pii/S1746809414000391

[15] S. Bhattacharyya, A. Konar, and D. N. Tibarewala, "Motor imagery, p300 and error-related eeg-based robot arm movement control for rehabilitation purpose," Med. Biol. Eng. Comput., vol. 52, no. 12, pp. 1007-1017, Dec. 2014.

[16] J. R. Millan, F. Renkens, J. Mourino, and W. Gerstner, "Noninvasive brain-actuated control of a mobile robot by human EEG," IEEE Trans. Biomed. Eng., vol. 51, no. 6, pp. 1026-1033, Jun. 2004.

[17] S. Bhattacharyya et al., "EEG controlled remote robotic system from motor imagery classification," in Proc. 3rd Int. Conf. Comput. Commun. Netw. Technol. (ICCCNT), Coimbatore, India, Jul. 2012, pp. 1-8.

[18] Y. Chae, J. Jeong, and S. Jo, "Toward brain-actuated humanoid robots: Asynchronous direct control using an EEG-based BCI," IEEE Trans. Robot., vol. 28, no. 5, pp. 1131-1144, Oct. 2012.

[19] J. Long et al., "A hybrid brain computer interface to control the direction and speed of a simulated or real wheelchair," IEEE Trans. Neural Syst. Rehabil. Eng., vol. 20, no. 5, pp. 720-729, Sep. 2012.

[20] D. Coyle, J. Garcia, A. R. Satti, and T. M. McGinnity, "EEG-based continuous control of a game using a 3 channel motor imagery BCI: BCI game," in Proc. IEEE Symp. Comput. Intell. Cogn. Algorithm. Mind Brain (CCMB), Paris, France, Apr. 2011, pp. 1-7.

[21] J. Li and L. Zhang, "Bilateral adaptation and neurofeedback for brain computer interface system," J. Neurosci. Methods, vol. 193, no. 2, pp. 373-379, 2010. [Online]. Available: http://www.sciencedirect.com/ science/article/pii/S0165027010005224

[22] J. Faller et al., "A co-adaptive brain-computer interface for end users with severe motor impairment," PLOS ONE, vol. 9, no. 7, 2013, Art. no. e101168.

[23] J. DiGiovanna, B. Mahmoudi, J. Fortes, J. C. Principe, and J. C. Sanchez, "Coadaptive brain-machine interface via reinforcement learning," IEEE Trans. Biomed. Eng., vol. 56, no. 1, pp. 54-64, Jan. 2009.

[24] M. J. Bryan, S. A. Martin, W. Cheung, and R. P. Rao, "Probabilistic co-adaptive brain-computer interfacing," J. Neural Eng., vol. 10, no. 6, 2013, Art. no. 066008. [Online]. Available: http://stacks.iop.org/1741-2552/10/i=6/a =066008

[25] F. Galan et al., "A brain-actuated wheelchair: Asynchronous and non-invasive brain-computer interfaces for continuous control of robots," Clin. Neurophysiol., vol. 119, no. 9, pp. 2159-2169, 2008. [Online]. Available: http://www.sciencedirect.com/ science/article/pii/S1388245708005750

[26] M. Hayashibe and S. Shimoda, "Synergetic motor control paradigm for optimizing energy efficiency of multijoint reaching via tacit learning," Front. Comput. Neurosci., vol. 8, no. 21, 2014, pp. 613-623. [Online]. Available: http://journal.frontiersin.org/article/10.3389/ fncom.2014.00021/full

[27] J. W. Krakauer, M. F. Ghilardi, and C. Ghez, "Independent learning of internal models for kinematic and dynamic control of reaching," Nat. Neurosci., vol. 2, no. 11, pp. 1026-1031, Nov. 1999.

[28] W. T. Thach, "What is the role of the cerebellum in motor learning and cognition?" Trends Cogn. Sci., vol. 2, no. 9, pp. 331-337, 1998. [Online]. Available: http://www.sciencedirect.com/ science/article/pii/S1364661398012236

[29] D. M. Wolpert, R. C. Miall, and M. Kawato, "Internal models in the cerebellum," Trends Cogn. Sci., vol. 2, no. 9, pp. 338-347, 1998. [Online]. Available: http://www.sciencedirect.com/ science/article/pii/S1364661398012212

[30] M. Kawato, "Internal models for motor control and trajectory planning," Current Opinion Neurobiol., vol. 9, no. 6, pp. 718-727, 1999.

[31] T. Flash and N. Hogan, "The coordination of arm movements: An experimentally confirmed mathematical model," J. Neurosci., vol. 5, no. 7, pp. 1688-1703, 1985.

[32] Y. Uno, M. Kawato, and R. Suzuki, "Formation and control of optimal trajectory in human multijoint arm movement. Minimum torque-change model," Biol. Cybern., vol. 61, no. 2, pp. 89-101, 1989. 
[33] C. M. Harris and D. M. Wolpert, "Signal-dependent noise determines motor planning," Nature, vol. 394, pp. 780-784, Aug. 1998.

[34] R. M. Alexander, "A minimum energy cost hypothesis for human arm trajectories," Biol. Cybern., vol. 76, no. 2, pp. 97-105, 1997.

[35] J. Nakanishi, R. Cory, M. Mistry, J. Peters, and S. Schaal, "Operational space control: A theoretical and empirical comparison," Int. J. Robot. Res., vol. 27, no. 6, pp. 737-757, Jun. 2008. [Online]. Available: http://dx.doi.org/10.1177/0278364908091463

[36] J. Peters and S. Schaal, "Learning to control in operational space," Int. J. Robot. Res., vol. 27, no. 2, pp. 197-212, 2008. [Online]. Available: http://www-clmc.usc.edu/publications/P/peters-IJRR2008.pdf

[37] S. Shimoda, Y. Yoshihara, and H. Kimura, "Adaptability of tacit learning in bipedal locomotion," IEEE Trans. Auton. Mental Develop., vol. 5, no. 2, pp. 152-161, Jun. 2013.

[38] S. Sanei and J. Chambers, EEG Signal Processing. Chichester, West Sussex, U.K.: Wiley-Intersci., 2007.

[39] D. J. Barrett and R. E. Silverman, SSH, The Secure Shell: The Definitive Guide. Cambridge, MA, USA: O'Reilly, 2001.

[40] S. Darvishi and A. Al-Ani, "Brain-computer interface analysis using continuous wavelet transform and adaptive neuro-fuzzy classifier," in Proc. IEEE 29th Annu. Int. Conf. Eng. Med. Biol. Soc. (EMBS), Aug. 2007, pp. 3220-3223.

[41] P. Herman, G. Prasad, T. M. McGinnity, and D. Coyle, "Comparative analysis of spectral approaches to feature extraction for EEG-based motor imagery classification," IEEE Trans. Neural Syst. Rehabil. Eng., vol. 16, no. 4, pp. 317-326, Aug. 2008.

[42] M. Belkin and P. Niyogi, "Laplacian Eigenmaps for dimensionality reduction and data representation," Neural Comput., vol. 15, no. 6, pp. 1373-1396, Jun. 2003. [Online]. Available: http://dx.doi.org/10.1162/089976603321780317

[43] D. J. Sebald and J. A. Bucklew, "Support vector machine techniques for nonlinear equalization," IEEE Trans. Signal Process., vol. 48, no. 11, pp. 3217-3226, Nov. 2000.

[44] C. Qiang, P. Hu, and F. Huanqing, "Experiment study of the relation between motion complexity and event-related desynchronization/synchronization," in Proc. 1st Int. Conf. Neural Interface Control, Wuhan, China, May 2005, pp. 14-16.

[45] S. Bhattacharyya, D. Basu, A. Konar, and D. N. Tibarewala, "Interval type-2 fuzzy logic based multiclass anfis algorithm for real-time EEG based movement control of a robot arm," Robot. Auton. Syst., vol. 68, pp. 104-115, Jun. 2015.

[46] I. Fodor, "A survey of dimension reduction techniques," Center Appl. Sci. Comput., Livermore Nat. Lab., Livermore, CA, USA, Tech. Rep. UCRL-ID-148494, 2002.

[47] L. van der Maaten, E. O. Postma, and H. J. van den Herik, "Dimensionality reduction: A comparative review," MICC, Maastricht Univ., Maastricht, The Netherlands, Tech. Rep. TiCC TR 2009-005, pp. 1-22, 2008.

[48] E. Bizzi, F. Mussa-Ivald, and S. Giszter, "Computations underlying the execution of movement: A biological perspective," Science, vol. 253, pp. 287-291, Jul. 1991.

[49] E. Alpaydin, Introduction to Machine Learning (Adaptive Computation and Machine Learning). Cambridge, MA, USA: MIT Press, 2004.

[50] J. A. Hanley and B. J. McNeil, "The meaning and use of the area under a receiver operating characteristic (roc) curve," Radiology, vol. 143, no. 1, pp. 29-36, 1982.

[51] M. Fatourechi et al., "Comparison of evaluation metrics in classification applications with imbalanced datasets," in Proc. 7th Int. Conf. Mach. Learn. Appl. ICMLA, San Diego, CA, USA, Dec. 2008, pp. 777-782.

[52] J. Hurtado-Rincon, S. Rojas-Jaramillo, Y. Ricardo-Cespedes, A. M. Alvarez-Meza, and G. Castellanos-Dominguez, "Motor imagery classification using feature relevance analysis: An Emotiv-based BCI system," in Proc. Symp. Image Signal Process. Artif. Vis. (STSIVA), Sep. 2014, pp. 1-5.

[53] S. Dharmasena, K. Lalitharathne, K. Dissanayake, A. Sampath, and A. Pasqual, "Online classification of imagined hand movement using a consumer grade EEG device," in Proc. 8th IEEE Int. Conf. Ind. Inf. Syst. (ICIIS), Peradeniya, Sri Lanka, Dec. 2013, pp. 537-541.

[54] S. Fok et al., "An EEG-based brain computer interface for rehabilitation and restoration of hand control following stroke using ipsilateral cortical physiology," in Proc. IEEE Annu. Int. Conf. Eng. Med. Biol. Soc. (EMBC), Boston, MA, USA, Aug./Sep. 2011, pp. 6277-6280.

[55] R. R. Bouckaert, "Choosing between two learning algorithms based on calibrated tests." in Proc. Mach. Learn. Int. Conf., Menlo Park, CA, USA, 2003, pp. 51-58.
[56] J. Faller et al., "Online co-adaptive brain-computer interfacing: Preliminary results in individuals with spinal cord injury," in Proc. 6th Int. IEEE/EMBS Conf. Neural Eng. (NER), San Diego, CA, USA, Nov. 2013, pp. 977-980.

[57] R. Kus et al., "Asynchronous BCI based on motor imagery with automated calibration and neurofeedback training," IEEE Trans. Neural Syst. Rehabil. Eng., vol. 20, no. 6, pp. 823-835, Nov. 2012.

[58] Z. Li, M. Hayashibe, C. Fattal, and D. Guiraud, "Muscle fatigue tracking with evoked EMG via recurrent neural network: Toward personalized neuroprosthetics," IEEE Comput. Intell. Mag., vol. 9, no. 2, pp. 38-46, May 2014.

[59] M. Hayashibe, D. Guiraud, J. L. Pons, and D. Farina, "Editorial: Biosignal processing and computational methods to enhance sensory motor neuroprosthetics," Front. Neurosci., vol. 9, no. 434, pp. 6-9, 2015. [Online]. Available: http://www.frontiersin.org/ neuroprosthetics/10.3389/fnins.2015.00434/full

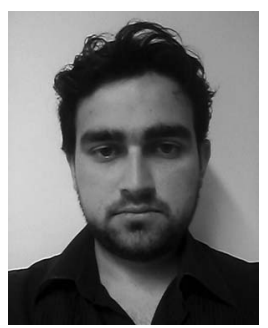

Saugat Bhattacharyya received the B.Tech. degree in biomedical engineering from the Siliguri Institute of Technology, Chamta, India, in 2009, and the M.E. and Ph.D. degrees in biomedical engineering from Jadavpur University, Kolkata, India, in 2011 and 2015 , respectively.

$\mathrm{He}$ is currently a Post-Doctoral Researcher with the BCI-LIFT Project, CAMIN Project Team, Institute National de Recherche en Informatique et en Automatique (INRIA), Montpellier, France. He did the Ph.D. internship in the DEMAR Project Team, INRIA, from 2014 to 2015, as part of the Erasmus Mundus-Svaagata Project Fellowship, in 2014. His current research interests include brainmachine interface, bio-potential signal processing, machine learning, rehabilitative engineering and robotics, biomedical image processing, biomedical instrumentation, and biomedical control systems.

Dr. Bhattacharyya was a recipient of the Council of Scientific and Industrial Research Senior Research Fellowship, India, in 2012.

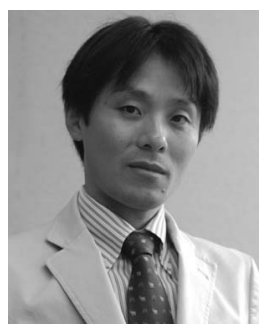

Shingo Shimoda received the B.S., M.S., and $\mathrm{Ph} . \mathrm{D}$. degrees in mechanical and electronic from the University of Tokyo, Tokyo, Japan, in 1999, 2001, and 2005, respectively.

He was a Research Scientist with the Biomimetic Control Research Center, RIKEN, Saitama, Japan, in 2005. In 2008, he became a Unit Leader with RIKEN Brain Research Institute-TOYOTA Collaboration Center, Intelligent Behavior Control Collaboration Unit, Nagoya, Japan.

Dr. Shimoda was a recipient of the General Chairs' Recognition Award in CDC 2009 and the CoTeSys Cognitive Robotics Best Paper Award in IROS 2010.

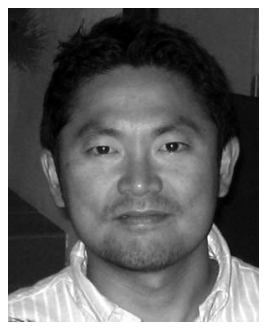

Mitsuhiro Hayashibe (M'04-SM'14) received the B.S. degree in mechano-aerospace engineering from Tokyo Institute of Technology, Tokyo, Japan, in 1999, and the M.S. and Ph.D. degrees from the Graduate School of Engineering, University of Tokyo, Tokyo, Japan, in 2001 and 2005, respectively.

$\mathrm{He}$ was an Assistant Professor with the Department of Medicine, Jikei University School of Medicine, Tokyo, Japan, from 2001 to 2006 . He was a Post-Doctoral Fellow with Institute National de Recherche en Informatique et en Automatique (INRIA), Sophia Antipolis and Laboratoire d'Informatique, de Robotique et de Microelectronique de Montpellier (LIRMM) of CNRS/University of Montpellier, Montpellier, France, in 2007. Since 2008, he has been a Tenured Research Scientist with INRIA and University of Montpellier, Computational Medicine and Neurosciences, Demar team. He has been a Visiting Researcher with RIKEN Brain Science Institute, and TOYOTA Collaboration Center, Nagoya, Japan, since 2012. His current research interests include personalized modeling for neuroprosthetics and neurorehabilitation.

Dr. Hayashibe was a recipient of the Best Paper Award from the Journal of Japanese Society for Computer-Aided Surgery, CAS Young Investigator Award, and the Gold Prize from Hitachi Medical Systems. He serves as an Editorial Board Member for medical robotics section of the International Journal of Advanced Robotic Systems and the Co-Chair of the IEEE Robotics and Automation Society Technical Committee on Human Movement Understanding. He is a Senior Member of the IEEE Engineering in Medicine and Biology Society. 